$40.5 \%$ as high risk, compared to $79.5 \%$ and $84.9 \%$ using the SIRS criteria and CURB65 respectively.

Conclusions All three scoring systems can stratify according to risk of 30 day mortality, though none of them are particularly accurate. qSOFA has poor sensitivity, and may underestimate severity and risk of 30 day mortality in CAP. New assessment tools to accurately identify CAP patients at increased risk of poor outcomes are urgently required.

\begin{tabular}{|c|c|c|c|c|c|c|}
\hline Score & $\begin{array}{l}\text { Sensitivity } \\
(\%)\end{array}$ & $\begin{array}{l}\text { Specificity } \\
(\%)\end{array}$ & $\begin{array}{l}\text { NPV } \\
(\%)\end{array}$ & $\begin{array}{l}\text { PPV } \\
(\%)\end{array}$ & $\begin{array}{l}\text { Negative } \\
\text { LR }\end{array}$ & $\begin{array}{l}\text { Positive } \\
\text { LR }\end{array}$ \\
\hline CURB65 & 84.9 & 40 & 91.5 & 25.9 & 0.38 & 1.42 \\
\hline SIRS & 79.5 & 32.5 & 87.3 & 21.4 & 0.63 & 1.18 \\
\hline qSOFA & 40.5 & 79.5 & 84.3 & 32.9 & 0.75 & 1.98 \\
\hline
\end{tabular}

NPV - negative predictive value, PPV - positive predictive value, LR - Likelihood ratio

\section{S9 $\quad$ INVASION VERSUS OVERGROWTH: UNDERSTANDING WHY RESPIRATORY PATHOGENS COLONISE THE MOUTH PRIOR TO DEVELOPMENT OF PNEUMONIA}

${ }^{1} \mathrm{VC}$ Ewan, ${ }^{1} \mathrm{~W}$ Reid, ${ }^{1} \mathrm{M}$ Shirley, ${ }^{2} \mathrm{~W}$ Wade, ${ }^{1} \mathrm{SP}$ Rushton, ${ }^{1} \mathrm{AJ}$ Simpson. ${ }^{1}$ Newcastle University, Newcastle upon Tyne, UK; ${ }^{2}$ Queen Mary University of London, London, UK

\subsection{6/thoraxjnl-2017-210983.15}

Introduction The presence of respiratory pathogens on oral surfaces is a risk factor for pneumonia. Understanding why non-oral respiratory pathogens appear is crucial in planning interventions to manipulate the oral microbiota to prevent pneumonia. We sought to understand whether respiratory pathogens were associated with reduction in oral bacterial diversity (invasion hypothesis) or no change in diversity (overgrowth hypothesis).

Methods We analysed extracted DNA from 167 throat samples from 53 hospitalised older patients with hip fracture using next generation sequencing (Lib-L chemistry, mothur). Occurrence of respiratory tract infection (RTI, clinician-initiated antibiotic for chest infection) within 3 months of discharge was noted via case notes and telephone call to General Practitioner. We used linear mixed effect modelling in $\mathrm{R}$ (nlme package) to investigate the association between relative abundance of respiratory pathogens and species richness, with patient as the random effect. We used correspondence analysis (CA) to analyse beta-diversity (vegan package).

Results Respiratory pathogens (Haemophilus influenzae, Staphylococcus aureus, Enterobactericeae) were present in $38 / 167$ samples (23\%). Higher relative abundances of respiratory pathogens were not significantly associated with sample diversity $(\mathrm{t}=-1.400575, \mathrm{p}=0.1641)$. Moreover, mixed effect models demonstrated no increase in relative abundances of respiratory pathogens over time in individual patients whilst in hospital $(\mathrm{t}=-0.206605, \mathrm{p}=0.8367)$. While RTI was associated with higher relative abundances of respiratory pathogens $(t=1.9502718, p=0.0567)$, there was no association between RTI and oropharyngeal species richness $(t=-0.361164$, $\mathrm{p}=0.7195$ ).

Conclusion There was no association between detection of respiratory pathogens and oropharyngeal species diversity. These Results support the overgrowth, rather than the invasion, hypothesis, and larger studies to explore frequency of oral clearance in conjunction with the oral microbiota are warranted. In addition, the lack of change over time in relative abundances of respiratory pathogens suggests that the exposure to the hospital environment is not a major driver in the appearance of these organisms.

\section{S10 SUPPRESSION OF MACROPHAGE INFLAMMATORY RESPONSES TO STREPTOCOCCUS PNEUMONIAE BY REGULATORY T CELLS}

G Szylar, J Brown. University College London, London, UK

\subsection{6/thoraxjnl-2017-210983.16}

Background The highly inflammatory immune response to Streptococcus pneumoniae infection can result in complications such as sepsis and Acute Respiratory Distress Syndrome. Macrophages are an important source of the inflammatory cytokines that activate epithelial and endothelial cells, resulting in a loss of barrier integrity. Regulatory T cells (Tregs) are a population of anti-inflammatory cells that modulate macrophage activity and are protective against invasive pneumococcal disease in mice. ${ }^{1,2}$

Aims To characterise the in vitro effects of Tregs on the macrophage inflammatory response to $S$. pneumoniae and to observe Treg recruitment to the site of intradermal injection of UV-killed S. pneumoniae in a human model.

Results Preliminary data suggest that co-culture of human monocyte-derived macrophages (MDMs) with $\mathrm{CD} 4{ }^{+} \mathrm{CD} 25^{+} \mathrm{CD} 127^{-}$Tregs reduced MDM TNF $\alpha$ production by at least $45 \%$ (One-way ANOVA $\mathrm{p}<0.01$ ) and IL-6 production by at least 52\% (One-way ANOVA p<0.01) 72 hours after initial infection with $S$. pneumoniae TIGR4 strain (MOI of 2, ratio of 1 Treg to $3 \mathrm{MDMs}$ ). Separation of Tregs from the MDMs during co-culture using transwell inserts prevented the suppressive effects of the Tregs. Using a novel human model of S. pneumoniae challenge involving intradermal injection of UV-killed S. pneumoniae into the forearm of healthy volunteers, we demonstrated that Tregs accumulated at the site of injection within 48 hours, increasing from undetectable Treg population at 4 hours to constituting approximately $33 \%$ of CD4 cells by 48 hours.

Conclusion Preliminary data suggest that Tregs modulate the MDM inflammatory response to $S$. pneumoniae in a contactdependent manner, and track to the site of intradermal injection of the UV-killed bacteria in human volunteers.

\section{REFERENCES}

1. Tiemessen MM, Jagger $A L$, Evans HG, van Herwijnen MJ, John S, Taams LS. CD4 +CD25+FoxP3+ regulatory $T$ cells induce alternative activation of human monocytes/macrophages. Proc Natl Acad Sci 2007;104(49):19446-51.

2. Neill DR, Fernandes VE, Wisby L, Haynes AR, Ferreira DM, Laher A, Strickland N, Gordon SB, Denny P, Kadioglu A, Andrew PW. T regulatory cells control susceptibility to invasive pneumococcal pneumonia in mice. PLoS Pathog 2012;8(4): e1002660. 